\title{
WILHELM GUgGENBERGER Die Maschine als besserer Mensch Zur Faszination technischer Perfektion
}

Die Forschung an Artificial Intelligence (AI) oder künstlicher Intelligenz (KI) mag als die Königsklasse der Informatik gelten. Zugleich erscheint sie aber auch als etwas anrüchig. Es haftet ihr ein Image an, das sie in die Nähe zur Alchemie rückt. Diese suchte in vergangener Zeit faszinierende Ziele zu erreichen, denen sich auch gesellschaftlich einflussreiche Persönlichkeiten verschrieben, musste aber dennoch im Verborgenen arbeiten, weil die Mittel zur Erreichung dieser Ziele als problematisch, an der Grenze des ethisch Vertretbaren stehend, ja gar als sakrilegisch galten.

Sowohl die hohen Erwartungen, die sich an die Entwicklung künstlicher Intelligenzen knüpfen, als auch die ambivalente Gefühlslage ihr gegenüber machen die Thematik zu einem beliebten Stoff für Science-Fiction, sowohl im Bereich der Literatur, als auch in jenem des Films. Das trägt dazu bei, dass das Thema als etwas wahrgenommen wird, das nicht so ganz in den Bereich faktenbasierter Wissenschaft gehört, sondern eher in den der Dichtung und der Fantasie. Dies ist wohl eine grobe Fehleinschätzung. Gerade die unterschiedlichen Darstellungen im Feld der Science-Fiction bieten hingegen die Gelegenheit nach den Hoffnungen zu fragen, die Einzelpersonen aber auch die Gesamtgesellschaft an KI knüpfen und was sie dazu bewegt, in diesen Forschungsbereich geistige Energie und finanzielle Mittel $\mathrm{zu}$ investieren. Ebenso wird daran sichtbar, welche Befürchtungen sich mit der Vorstellung verbinden, diese Forschung könnte erfolgreich sein. Im Folgenden soll gerade dieser Frage nach Erwartungen und Befürchtungen vor dem Hintergrund eines christlich geprägten Menschenbildes nachgegangen werden. 


\section{$1 \mathrm{KI}$ - was ist das?}

Die Potentiale künstlicher Intelligenz faszinieren und eröffnen ein Feld schier unbegrenzter Möglichkeiten. Vielerlei Gegenstände werden bereits heute mit dem Adjektiv smart, also schlau, klug oder eben intelligent ausgezeichnet. Das gilt für Mobiltelefone ebenso, wie für Häuser, Stromnetze oder ganze Städte. Mit welcher Begründung nennen wir derartige Artefakte, die Produkte menschlicher Herstellung und letztlich doch nur Werkzeuge sind, schlau? Was wir von ihnen erwarten und was sie zumindest teilweise schon zu leisten in der Lage sind, ist die Kontrolle vorgegebener Zielparameter, die selbst im Blick zu behalten für uns sehr mühsam wäre, weil dafür eine Vielzahl an Faktoren oder Randbedingungen berücksichtigt werden muss. Ein Smart-Grid, ein schlaues Stromnetz z. B. soll die verfügbare Energie so auf Regionen, Unternehmen und Haushalte verteilen, dass die Auslastung des Netzes immer optimal ist und auch die Kosten für Abnehmer möglichst niedrig bleiben. Im Haushalt kann man dann etwa die Waschmaschine befüllen und den Startknopf drücken; wann das Gerät tatsächlich zu waschen beginnt, würde jedoch vom Netzwerk danach entschieden, wann der Stromtarif am günstigsten ist, was wiederum davon abhängt, ob hunderte Kilometer entfernt gerade Wind weht, um Turbinen anzutreiben oder wie viel elektrische Energie derzeit nachgefragt wird. Man mag sich auch bereits darauf verlassen, dass das eigene Fahrzeug so schlau ist, die Scheinwerfer abhängig von der Umgebungshelligkeit selbsttätig ein- und auszuschalten, das Fernlicht abzublenden, wenn ein anderes Fahrzeug entgegenkommt, oder über das Navigationssystem eine alternative Strecke zu wählen, weil auf der gewohnten Route gerade Stau herrscht.

Vieles von dem ließe sich auch ganz ohne smarte Technologie erledigen, geht mit ihr aber eben bequemer. Es gilt hier, was sich von Technik ganz allgemein sagen lässt, dass sie einer Anstrengung entspringt, die dazu dient, Anstrengungen zu vermeiden. ${ }^{1}$ Die durchaus

1 Ortega y Gasset (1956), 3: „Halten wir daher fest, dass die Technik zunächst die Anstrengung ist, Anstrengung zu sparen, oder, anders ausgedrückt, sie ist das, 
beachtliche Leistungsfähigkeit von smarten Geräten hat letztlich aber wenig mit Intelligenz zu tun, auch wenn man zugeben muss, dass es keine allgemein anerkannte Definition dafür gibt, was Intelligenz überhaupt sei. Die Funktionalität der Geräte beruht auf Entscheidungsregeln, die durch die Programmierung der verbauten Prozessoren festgelegt sind, sowie auf hoher und schneller Rechenleistung. Auf diese Weise können enorme Datenmengen in Entscheidungsprozesse einbezogen werden, wie eben z. B. Informationen über die Einspeisung von Strom aus vielfältigen Quellen in ein Energienetz, über die momentane Nachfrage durch eine noch viel größere Anzahl von Endabnehmern und über eine festgelegte Präferenzordnung dieser Abnehmer, die regelt, wer zuerst beliefert werden soll. Dies durch menschliche Hand- oder Kopfarbeit erledigen zu lassen, würde viel Zeit beanspruchen und könnte damit den angestrebten Zweck kaum erfüllen. Dennoch handelt es sich dabei um eine Leistung, die qualitativ jener eines simplen Thermostaten entspricht: die Optimierung eines Zustandes nach vorgegebenen Maßstäben durch die Verarbeitung von Messdaten. Angesichts der mittlerweile verfügbaren Datenmengen (big data $)^{2}$, aus denen sich durch regelgeleitete Verknüpfungen Schlüsse ziehen lassen, wird diese Form der KI immer bedeutsamer. An sie knüpfen sich auch zahlreiche ethische Fragen, die mit Datenschutz, der monopolistischen Konzentration von Schaltstellen und der Verfügung über Datenschätze, auch mit der mehr oder weniger subtilen Manipulation von AkteurInnen durch die Art, wie diese Da-

was wir tun, um ganz oder teilweise das zu vermeiden, was unsere Umwelt uns in erster Linie zu tun auferlegt."“

2 Als VerwenderInnen von Alltagsinformatik waren wir es vor wenigen Jahrzenten gewohnt in Kilobyte zu rechnen $\left(1000=10^{3}\right.$ Byte), heute hantieren wir vielfach mit Datenträgern im Hosentaschenformet, die zweistellige Gigabyte-Datenmengen $\left(10^{9}\right.$ Byte) speichern können. Die weltweiten Datenmengen werden mit etwa 40.000 Exabyte $\left(10^{18}\right.$ Byte) angegeben, wobei nicht nur Inhalte von Interesse sind, sondern insbesondere Metadaten über Nutzung und Nutzer der Inhalte. Vgl Kreutzer / Sirrenberg (2019), 80-81. 
ten wieder zugänglich gemacht werden, zu tun haben. Dies soll hier nicht im Zentrum meiner Überlegungen stehen.

Was an dieser Stelle interessiert, ist das Streben nach jenem qualitativen Sprung, der den Punkt übersteigt, den InformatikerInnen als Singularität bezeichnen. Singularität meint in diesem Fall den Moment, in dem die Intelligenz einer Maschine jener des Menschen gleichzusetzen wäre. So weit sind wir noch nicht, auch wenn es mitunter anders scheinen mag. Dass Computer die besten Schachspieler oder Go-Meister zu schlagen vermögen, kann unsere Wahrnehmung in die Irre führen. Wir sind durch diese Leistungen beeindruckt; dennoch handelt es sich dabei noch immer um quantitativ gesteigerte Rechenleistung. Viel schwieriger, als dem Gerät die Regeln eines Spiels und deren Handhabung beizubringen, ist etwa, es erkennen zu lassen, dass erwachsene Personen, die in einem Park unförmige Plastikteile umhertragen, Schach spielen, ein Kind, das dieselben Objekte hin und her bewegt, aber nicht. Die Wahrnehmung und Interpretation von Alltagsbildern, die sich dem Menschen in Sekundenbruchteilen in ihrer Bedeutung erschließen, stellt für die KI-Forschung eine wesentlich größere Herausforderung dar, als es jene der Entwicklung von Schachcomputern war. Ein autonomes Fahrzeug müsste aber freilich auch dazu in der Lage sein, sollen wir ihm über den Weg trauen können.

Die Fortschritte, die auf diesem Feld laufend erzielt werden, beruhen darauf, dass Programme heute fähig sind, selbst zu lernen. Sie nützen dabei die enormen Datenmengen im globalen Netz, und erkennen Muster im Verhalten von Usern. Daraus leiten sie Prognosen für die Zukunft ab. So kann ein Programm etwa lernen, dass es in der Kommunikation mit Menschen hilfreich sein dürfte, Bilder von niedlichen Katzen zu posten, ohne deshalb auch schon tierlieb zu sein, oder es kann lernen, dass sich viele Follower durch sexistische oder fremdenfeindliche Aussagen gewinnen lassen, ohne erfassen zu müssen, was solche Aussagen bedeuten. Selbst wenn wir es bislang also noch immer nur mit dem Sammeln und Prozessieren großer Datenmengen zu 
tun haben, lässt sich doch vermuten, dass sich auf dem eingeschlagenen Weg irgendwann auch eigenständig kreatives Lernen entwickeln könnte; so etwas wie Phantasie oder eine freie Stellungnahme zu den bearbeiteten Daten und damit Geist, Bewusstsein, ja sogar Selbstbewusstsein. Die Einschätzungen darüber, ob, und wenn ja, wann dies der Fall sein wird, gehen stark auseinander. Einigkeit besteht jedoch weitgehend darüber, dass maschinelle Intelligenz - wäre ein solcher Singularitätspunkt erst einmal erreicht - die menschliche rasch und deutlich überflügeln dürfte.

\section{Wer will denn so etwas?}

Hinsichtlich der technischen Details, die für die Entwicklung einer solch starken künstlichen Intelligenz vorauszusetzen sind, besitzt ein Theologe keine Kompetenz. Ich vermag auch nicht zu entscheiden, ob die Bemühungen darum realistischer Weise erfolgversprechend sind. Mir genügt jedoch die Feststellung, dass es diese Bemühungen gibt und dass nicht wenige hochbegabte und hochdotierte WissenschaftlerInnen daran arbeiten, sie zu einem Erfolg zu führen, um die Frage zu stellen, warum wir als menschliche Spezies an der Hervorbringung einer solchen Intelligenz interessiert sein sollten. Rufen wir damit nicht wie Goethes Zauberlehrling ein Geschöpf ins Dasein, das uns am Ende als Ausgeburt der Hölle erscheinen könnte, die nicht mehr auf uns hören will? Die mögliche Unfolgsamkeit solcher Konstrukte wäre in unserem Fall dann aber nicht durch einen vermeidbaren Fehler verursacht, sondern von Anfang an bewusst intendiert. Denn was wir uns von wahrer Intelligenz erwarten, ist ja gerade nicht penible Ausführung kleinteiliger Befehle, sondern die selbständige Bearbeitung komplexer Problemstellungen. Wollen wir dies ermöglichen, kann nicht zugleich garantiert werden, dass die konkrete Ausführung von Problemlösungen so erfolgen wird, wie wir uns das vorgestellt hatten. Oder noch deutlicher auf den Punkt gebracht: Wer autonom 
agierende und entscheidende Maschinen baut, darf sich hinterher nicht darüber wundern, dass diese sich seiner Kontrolle entziehen. ${ }^{3}$

Auch im vollen Bewusstsein dieser Tatsache wird jedoch an der Weiterentwicklung starker KI gearbeitet. Die damit verbundenen Visionen lassen sich grob in drei Kategorien einteilen, die sich nach dem Grad der Abgabe menschlicher Kompetenz und Entscheidungsmacht an die maschinelle Welt unterscheiden. Ihnen allen gemeinsam ist als treibende Motivation eine gewisse Defiziterfahrung, die sich mit dem Menschsein verbindet: Unsere Spezies erscheint als fehlerhaft und daher wenig zukunftstauglich, weswegen von der Maschine erhofft wird, sie möge einen besseren Menschen ermöglichen, oder gar als bessere Alternative an dessen Stelle treten.

Der bekannte Schriftsteller Daniel Kehlmann hat diesen Gedanken vorsichtig zum Ausdruck gebracht, nachdem er 2020 die Gelegenheit hatte, mit einem Computerprogramm zu interagieren, das mit ihm einen Text verfassen sollte; nicht als Schreibtool, sondern als gleichberechtigter Coautor. ${ }^{4}$ Kehlmann reflektiert über die Vorstellung, intelligente Maschinen könnten uns eines Tages als Krone der Schöpfung ablösen.

Der Gedanke an diese möglichen Nachfolger erfüllt mich weder mit Sorge, noch mit Angst. So vieles haben wir wirklich nicht gut gemacht - die Ungerechtigkeit unseres Wirtschaftssystems, der Hunger, die Armut, der tägliche Massenmord an den Tieren in unseren Schlachtfabriken, die Zerstörung des Weltklimas, von der wir alle wissen, ohne sie zu verhindern ... Die Idee, dass unsere Nachfolge-Intelligenzen vernünftiger agieren werden als wir, könnte einen auch beruhigen. ${ }^{5}$

Der Autor bleibt aber dennoch skeptisch, indem er fortsetzt:

Auch wenn ich den Gedanken nicht abscheulich finde, weiß ich dennoch, dass ich diese Zukunft auch dann nicht erleben wollte, wenn ich die Wahl

3 Vgl. Dupuy (2007), 239-240.

4 Das Experiment ist weitgehend gescheitert. Die Texte, in denen die beiden „Autoren" die Sätze des jeweils anderen aufgreifend, diese fortschreiben sollten, endeten regelmäßig im Absturz des Programms.

5 Kehlmann (2021), 19-20. 
hätte, sie mit einer Zeitmaschine aufzusuchen. Denn ich weiß ja nicht, ob die künftigen siliziumbasierten Intelligenzen noch Musik hören oder Gedichte lesen werden; ich vermute, das werden sie eher nicht tun, warum sollten sie! Die kommende Welt wäre wohl eine ohne Shakespeare und Bach, ohne Goethe und García Marquez und Miles Davis, und obwohl ich begreife, dass dagegen unter kosmologischen Gesichtspunkten wenig einzuwenden ist, verzichte ich doch dankend. ${ }^{6}$

Ein solch dankender Verzicht findet sich bei den Denkern, die ich nun als Repräsentanten der genannten Kategorien kurz und dadurch wohl auch verkürzt vorstellen möchte, nicht. Der Verweis auf einen drohenden Verlust menschlicher Kultur erscheint ihnen entweder als nicht plausibel oder aber als nicht relevant.

\subsection{Zunehmende Delegation von Entscheidungen}

Der in den letzten Jahren zu einiger Bekanntheit gelangte israelische Historiker Yuval Noah Harari, denkt in seinem Buch 21 Lektionen für das 21. Jahrhundert über Herausforderungen einer weiteren Entwicklung der Menschheit nach. Neben Themen der Politik fordern uns gemäß Harari derzeit besonders jene der Technologie, die uns auch vor die Frage stellt, ob wir unsere Freiheit nicht weitestgehend aufgeben sollten, weil diese ohnedies eine Illusion darstellt. Die Illusion der Freiheit besteht in der Überzeugung, rationale Entscheidungen $\mathrm{zu}$ treffen, während wir vielfach gefühlsgesteuert agieren. Gefühle wiederum seien nichts weiter als biochemische Prozesse. Harari setzt diese Prozesse mit Algorithmen gleich, die im Laufe der biologischen Evolution zu immer weiter verfeinerten Programmen weiterentwickelt wurden. Wenn wir den Menschen also letztlich nur als biologische Maschine wahrnehmen, spricht nicht viel dagegen, dass er seine Entscheidungen an technische Maschinen delegiert, sofern diese über bessere Rechnerleistung verfügen. Wir sollten Harari zufolge also noch viel intensiver tun, was ohnedies bereits in vielen Bereichen geschieht: wir sollten maschinellen Algorithmen vertrau-

6 Kehlmann (2021), 19-20. 
en. „Schon heute definiert sich ,Wahrheit' anhand der Topresultate der Google-Suche. ${ }^{\text {"7 }}$ Dies gilt zumindest insofern, als in unserer Weltwahrnehmung faktisch nicht mehr auftaucht, was in den Suchergebnissen weiter hinten gereiht wird. Das personalisierte Programm entscheidet dabei gemäß meiner Interessen und Vorlieben, die es aus meinem bisherigen Verhalten errechnet hat. Kombinieren wir diese Technologie mit biometrischen Sensoren, die eine Vielzahl unserer Körperreaktionen überwachen, kennt uns der Algorithmus sehr bald besser, als wir uns selbst kennen. Dann, so der Autor, wäre es geradezu unverantwortlich neben der Auswahl von Filmen oder Büchern nicht auch Entscheidungen über die Berufswahl oder bezüglich des Partners / der Partnerin fürs Leben der Maschine zu überlassen.

Wenn sich die Macht von Menschen auf die Algorithmen verschiebt, sehen wir die Welt eventuell nicht mehr als Spielfeld autonomer Individuen, die darum ringen, die richtigen Entscheidungen zu treffen. Stattdessen betrachten wir das gesamte Universum vielleicht als einen Datenstrom, sehen in Organismen kaum mehr als biochemische Algorithmen und glauben, dass die kosmische Berufung der Menschheit darin besteht, ein allumfassendes Datenverarbeitungssystem zu schaffen - und dann darin aufzugehen. ${ }^{8}$

Dieses Zitat, in dem das „,vielleicht“ durchaus ernst genommen werden sollte, weil Harari einer solchen Zukunft doch nicht wirklich traut, ${ }^{9}$ führt bereits zu einem weiteren Autor hin und damit zur zweiten Kategorie der Vorstellung von maschineller Verbesserung des Menschen.

\footnotetext{
Harari (2018), 87.

8 Harari (2018), 90.

9 Deutlicher wird dieses Misstrauen in Hararis Buch Homo Deus. Die Herrschaft technischer Intelligenz über den Homo Sapiens, die dort dargestellt wird, kann als Dystopie gelesen werden, scheint aber doch unaufhaltsam, weil KI als Fortführung der bisher dominierenden biochemischen Algorithmen gesehen wird, s. Hararai (2017).
} 


\subsection{Koevolution von Mensch und Maschine}

Der italienische Philosoph Luciano Floridi sieht in der zunehmenden Digitalisierung und Robotisierung unserer Lebenswelt eine Schule der Demut.

Intelligenz war, und ist nach wie vor, eine ziemlich unbestimmte Eigenschaft, schwer zu definieren, doch wir waren uns sicher, dass kein anderes Geschöpf auf Erden uns mit seinen Gaben überflügeln konnte. ${ }^{10}$

Mittlerweile aber müssen wir, so Floridi, akzeptieren, dass es andere intelligente Wesen in dieser Welt gibt, wodurch ein überzogener Anthropozentrismus in Frage gestellt werde. Als Menschen haben wir uns somit in eine umfassende Infosphäre einzufügen, die die Biosphäre an Bedeutung zusehends übertrifft, aber so wie diese von anderen Existenzformen bevölkert wird, die unseren Respekt fordern. Künstliche und menschliche Akteure gehören dabei unterschiedlichen Welten an. ${ }^{11}$ KI kann ausschließlich vorgegebene Daten nach bestimmten Mustern systematisieren. Für Menschen hingegen gibt es keine uninterpretierten Daten. Wir verbinden jede Information mit einem spezifischen Sinn. Gemäß Floridi wird diese Differenz auch in Zukunft bestehen bleiben.

Intelligente Maschinen und Roboter machen Menschen somit nicht überflüssig. Auch können weder Menschen noch Maschinen die physische Welt als Möglichkeitsbedingung hinter sich lassen, existieren aber doch immer mehr in der digitalen Welt der Daten, in der Prozessoren mit enormer Rechnerleistung sich als überaus effizient, erfolgreich und somit menschlicher Vernunft überlegen erweisen. Die Infosphäre zwingt uns daher in starkem Ausmaß ihre Gesetze auf, was zu einer Koevolution von Maschine und Mensch führen wird.

Dies lässt sich etwa am Beispiel autonomer Fahrzeuge darlegen, die zunächst ein praktisches Werkzeug für Menschen darstellen. Ihr Funktionieren erfordert jedoch die fortschreitende Umgestaltung un-

\footnotetext{
Floridi (2015), 126.

1 Floridi (2015), 184.
} 
serer Lebenswelt zur Infosphäre, zu einem Raum, in dem an unendlich vielen Stellen Daten produziert und ausgetauscht werden (Satelliten, Verkehrskameras, Mobiltelefone zahlloser PassantInnen). Von Menschen gesteuerte Fahrzeuge stellen in dieser Umwelt ein unberechenbares Störfeuer dar und werden daher wohl nicht lange geduldet werden. Letztlich könnte jeder biologische Körper, der sich durch den digitalisierten Raum bewegt, nur noch als Fehlerquelle wahrgenommen werden. Das reibungslosere Funktionieren der Maschine zwingt uns ihre Gesetzmäßigkeiten auf und nötigt, diesen gemäß zu agieren. Dieses Phänomen ist keineswegs neu und lässt sich an jeder Form von Technologie aufzeigen. Man denke nur an automobile Fortbewegung im Allgemeinen, um die herum wir seit geraumer Zeit unsere Städte und Dörfer errichten. Sofern Informationstechnologie aber nicht nur physische Überlegenheit zeigt, wie dies bei mechanischer Technologie der Fall ist, sondern auch intellektuelle, wird ihre Dominanz der menschlichen Spezies weitaus größere Anpassungsbereitschaft abverlangen.

\subsection{Weitergabe der Evolutionsstafette (Transhumanismus)}

Warum sollte uns angesichts all dessen der Fortbestand der biologisch beschränkten Gattung Mensch überhaupt noch ein Anliegen sein? Diese Frage stellt sich der Softwarepionier und Direktor der Forschungsabteilung von Google, Ray Kurzweil. Kurzweil ist Transhumanist. Als solcher geht er davon aus, dass die bisherige biochemische Evolution des Lebens mit der menschlichen Spezies an einen Höhepunkt gelangt ist, der zugleich eine Sackgasse darstellt. Unsere Gattung hat aber eine Intelligenz geschaffen, die nicht nur unsere eigene übertreffen könnte, der es vielmehr auch gelingen könnte, unabhängig von dem doch recht störanfälligen und kurzlebigen biologischen Substrat zu werden, an das unser menschlicher Geist gebunden bleibt. ${ }^{12}$ Warum sollte man sich also nicht damit abfinden, dass Evolu-

12 Eigentlich gilt vorsichtiger formuliert, dass das, was wir Geist nennen, intersubjektiv nur zugänglich ist, sofern es an ein körperliches Substrat gebunden ist. 
tion nun auf einer neuen, höheren Stufe fortgesetzt wird, jenseits der menschlichen Gattung? Wenn Maschinen erst einmal den Punkt der Singularität überschritten haben, werden wir nicht mehr viel Energie darauf verschwenden, unsere physische Existenz mit technischen Mitteln zu verbessern, wie das im sogenannten Human Enhancement mit Hilfe von Nanotechnologie, Bio- und Gentechnologie in Zusammenarbeit mit Informationstechnologie und Kognitionswissenschaft versucht wird. Vielmehr sollten wir unseren Geist den Maschinen überantworten, die ihn weitertragen und fortentwickeln werden.

Zwischen Maschine und Mensch wird es keine klaren Unterschiede mehr geben. Die Maschinen werden uns davon überzeugen, dass sie ein Bewusstsein haben. Alle subtilen Regungen, die wir mit Bewusstsein assoziieren, werden in ihnen vorkommen. ${ }^{13}$

Als Menschheit mögen wir auf diese Weise vielleicht aus dem Universum verschwinden; was erhalten bleibt, sind Muster (patterns) von Bewusstsein und Intelligenz. ${ }^{14}$ Die Evolution, der wir bislang weitgehend machtlos ausgeliefert waren, würde so gleichermaßen für einen kurzen historischen Moment menschlicher Gestaltungsmacht unterworfen, nur um durch diese in die Sphäre der Technologie transformiert, bald wieder ihrer autonomen Bahn zu folgen.

\section{Weil Schwächen nicht liebenswert sind}

Die bisherige Analyse lässt klar erkennen, dass hinter den Visionen einer starken KI die Sehnsucht nach Überwindung menschlicher Schwächen steht, die als liebenswert zu empfinden wir keineswegs bereit sind. Solche Schwächen begegnen uns zumindest in drei unterschiedlichen Ausprägungen: intellektuell, physisch, moralisch.

Zweifellos kämpfen wir als Menschen mit unseren intellektuellen Schwächen. Gerade im akademischen Kontext ist dies immer wieder Anlass für Selbstzweifel aber auch für Rivalität. Dennoch scheinen die meisten Menschen von dieser Schwäche weit weniger geplagt

\footnotetext{
13 Kurzweil (2001), 108.

14 Vgl. Kurzweil (2005), 388.
} 
als von körperlicher Hinfälligkeit und moralischem Versagen. Unterschiede hinsichtlich Merkfähigkeit und kalkulatorischer Intelligenz sind leichter akzeptierbar als solche in anderen Lebensbereichen. So ist es auch kaum noch eine Diskussion wert, dass Computer uns in der Fähigkeit des Speicherns und Verarbeitens von Informationen bereits weit überlegen sind. Ich erlaube mir daher, diese Schwäche links liegen zu lassen und mich den beiden anderen zuzuwenden.

Die physische Schwäche menschlicher Existenz zeigt sich darin, dass wir anderen Lebewesen in vielen Belangen unterlegen sind. Sich mit Hilfe von Ultraschall im Dunkeln zu orientieren oder mehrstündige Tauchgänge zu unternehmen stellt für diverse tierische Spezies kein Problem dar, ist Menschen ohne technische Hilfsmittel aber unmöglich. Über diese Tatsache hinaus teilt jedoch jede Form biologischer Existenz die deutlichste und grundlegendste Schwäche mit uns: Sterblichkeit.

Maschinen ermüden und erkranken nicht, sie sterben auch nicht. Freilich können sie kaputtgehen. Folgen wir Kurzweil, ist dies jedoch nicht mit Tod gleichzusetzen, denn die maßgeblichen Informationsmuster können von Datenträger zu Datenträger weitergegeben werden. Hardware mag verschrottet werden, Software lässt sich kopieren und sichern. Kurzweil hofft so auch als Individuum zu überdauern, geht er doch davon aus, dass menschliches Bewusstsein sich in absehbarer Zeit auf Datenträger downloaden lässt. Bis dorthin versucht er mit Hilfe eines optimierten Lebensstils und unzähliger Nahrungsergänzungsmittel und Medikamente in seinem biologischen Körper zu überleben, den er bald nicht mehr zu brauchen hofft. KI verheißt somit die Überwindung des Todes. Fraglich ist, ob in dieser Form von Existenz Vorstellungen von Individualität oder Personalität noch sinnvoll verortet werden können, doch selbst deren Aufgabe mag im Sinn einer universalen Verschmelzung des Partikulären als Gewinn erscheinen.

So sehr unsere Sterblichkeit und insbesondere der Tod geliebter Mitmenschen eine Quelle der Beunruhigung und des Leids sind, so 
wenig sollte die Frage moralischer Schwäche als Antrieb menschlichen Handelns unterschätzt werden. Wir sind in vieler Hinsicht unzuverlässige Wesen, deren Geist zwar willig, in seiner derzeitigen Ausführung jedoch ausgesprochen schwach ist, was wir für gewöhnlich auf das Fleisch, also die Leiblichkeit des Menschseins zurückführen.

Auf unsere sittliche Unzuverlässigkeit nimmt auch Harari Bezug, wenn er auf die Entwicklung autonomer Fahrzeuge zu sprechen kommt. Diese könnten das fahrerische Können Michael Schumachers mit der ethischen Reife Immanuel Kants kombinieren, ohne dabei jemals müde oder abgelenkt zu sein und ohne im Konfliktfall doch den eigenen Vorteil über jenen anderer VerkehrsteilnehmerInnen zu stellen. Theoretisch stimmen die meisten Menschen der Überlegung $\mathrm{zu}$, ein Auto solle etwa im Fall des Bremsversagens lieber den Tod seiner Insassen in Kauf nehmen, als in eine Gruppe spielender Kinder zu rasen. Fragt man danach, wer bereit sei, ein solches Auto zu erwerben, schwindet die Begeisterung deutlich. ${ }^{15}$ Wie man selbst in einer konkreten Dilemmasituation, in der innerhalb von Sekundenbruchteilen reagiert werden muss, entscheiden würde, mag der eigenen Imagination überlassen bleiben. Emotionslos kalkulierende künstliche Intelligenz würde jedenfalls verlässlich gültigen Normen folgen, unabhängig von individuellen Vorlieben oder momentanen Stimmungsschwankungen.

Auch dieser Gedanke einer Überwindung sittlicher Hinfälligkeit begleitet die Entwicklung von Technologien seit geraumer Zeit. So schrieb etwa der Erfinder des Dampfhammers, James Nasmyth, in der zweiten Hälfte des 19. Jahrhunderts in Begeisterung über seine Maschinen: „Sie sind nie betrunken, fehlen nie bei der Arbeit, streiken nicht, sind zuverlässig in der Genauigkeit und Regelmäßigkeit des Arbeitens. " ${ }^{16}$ Wäre angesichts der Präzision und Verlässlichkeit, mit der schon einfache mechanische Maschinen arbeiten, nicht zu erwarten, dass viel weiter entwickelte Maschinen auch in Fragen des

\footnotetext{
15 Vgl. Harari (2018), 96-97.

16 Zitiert nach Metz (2006), 162.
} 
gesellschaftlichen Zusammenlebens, in denen zwischen unterschiedlichsten Interessen abzuwägen und komplexe Konstellationen auf ein gemeinsames Wohl hin zu optimieren sind, zu besseren und gerechteren Lösungen kämen. Endlich wäre so das Ideal einer reinen Vernunft realisierbar, die frei vom emotional-triebhaften Erbe zu werden vermag, das wir aus unserer biologischen Stammesgeschichte mitschleppen.

So können wir festhalten, dass von uns konstruierte Geräte, insbesondere in ihrer informationstechnologischen Ausformung, in der Lage zu sein scheinen, einige der zentralsten Probleme unseres Daseins zu lösen, indem sie uns unsterblich und in einer solch imaginierten infiniten Existenz auch noch moralischer zu machen versprechen. ${ }^{17}$

\section{Die Scham der Geborenen}

Wenn uns angesichts solcher Verheißungen ein gewisses Unbehagen beschleicht, mag es an dem liegen, was der Philosoph Günther Anders als prometheische Scham bezeichnet hat. Der Titan Prometheus gilt in der griechischen Mythologie als Schöpfer des Menschen. Gegen den Willen des olympischen Göttervaters Zeus lässt er den Menschen auch die Gabe des Feuers zukommen, wofür dieser ihn grausam bestraft. Insbesondere im neuzeitlichen Denken wurde Prometheus zum Inbegriff des Drangs nach Fortschritt und technischer Entwicklung, die sich durch überkommene Autoritäten und religiöse Tabus nicht bremsen lässt. Doch bei Anders zeichnet sich der prometheische Mensch nicht durch den mitunter auch trotzigen Stolz auf das, was er geschaffen hat, aus, sondern durch seine Scham darüber, im Vergleich mit seinen Produkten nicht bestehen zu können.

Dieser Gedanke kam dem Philosophen, als er in den 1940erJahren einen Bekannten beobachtete, der auf einer Ausstellung für

17 Vgl. Benedikter (2021), Position 2966: „Technology [...] will solve the most problems, and ultimately make humanity become both moral and 'immortal'." Siehe auch Dupuy (2015), 15. 
modernste Maschinen zu verstummen begann und offenkundig verschämt seine Hände hinter dem Rücken oder in den Taschen verbarg; Hände, die mit der Präzision der gezeigten Geräte nicht mithalten konnten. Dies sei nicht Ausdruck einer Verdinglichung des Menschen durch andere, etwa im Sinn der von Marx beklagten Reduktion der Arbeiterschaft auf ihre Produktionsfunktion. Vielmehr schäme sich hier ein Mensch dafür, kein Ding zu sein. „Eingeschüchtert durch die Überlegenheit und die Übermacht der Produkte, ist er bereits in deren Lager desertiert [...]: er verachtet sich nun so, wie die Dinge, wenn sie es könnten, ihn verachten würden." ${ }^{18}$ Die Kreatürlichkeit selbst, die Tatsache, geboren und nicht hergestellt zu sein, scheint zum Problem geworden, wodurch man der Unplanbarkeit, dem Zufall und den Mucken der Biologie ausgeliefert bleibt. Angesichts der genannten Visionen im Bereich von KI klingt es geradezu prophetisch, wenn Anders in poetischer Sprache festhält, dass wir wohl bereit wären, jedes Opfer zu bringen, könnten wir nur ,als Prothesen mit Prothesen in vertrautestem Verband"19 existieren.

Das Merkwürdige an solchen Beobachtungen ist, dass die menschliche Spezies mit großem Aufwand daran zu arbeiten scheint, sich selbst zu erübrigen oder loszuwerden. ${ }^{20}$ Man kann darin eine Analogie zu jener Verzweiflung sehen, die nach Sören Kierkegaard in Angst wurzelt. Der angstgetriebene Mensch will verzweifelt er selbst sein, sich keinesfalls verlieren. Zugleich will er aber auch verzweifelt nicht er selbst sein. Er will nämlich nicht derjenige sein, der er oder sie wirklich ist, sondern gleichsam ein phantastisches Idealbild seiner selbst. ${ }^{21}$ Die Verzweiflung entsteht nun gerade daraus, dass der Mensch in Momenten der Angst nicht bereit ist, seine existentielle Abhängigkeit zu akzeptieren, und daher nach dem Strohhalm der Selbstabsicherung greift. Das ist gleichbedeutend mit der Zurückweisung Gottes, dem

\footnotetext{
18 Anders (1968), 30.

19 Anders (1968), 36.

20 Vgl. Anders (1968), 90-91.

${ }^{21}$ Vgl. Kierkegaard (1983), 8-17.
} 
dieser Mensch sich nicht anvertrauen will. Wie aber kann es gelingen, in einer Situation des Bedroht- und Überfordertseins sich selbst als Garanten der Rettung und letzten Hort der Zuflucht zu behaupten? Einigermaßen glaubwürdig kann dies nur erscheinen, wenn sich das Rettende als eigenständige Identität präsentiert, wenn der verzweifelt nach Halt Suchende die Mittel seiner Selbstrettung von sich abzuspalten und zu verobjektivieren vermag. ${ }^{22}$ Dann nämlich kann zumindest vorübergehend das paradoxe Bild eines Münchhausen, der sich am eigenen Schopf aus dem Sumpf zieht, verschleiert werden hinter dem Bild eines schöpferischen Wesens, das sich durch seine Produkte aus dem Sumpf ziehen lässt. Diesen selbstkonstruierten Rettern unterwerfen wir uns gleichsam als unseren Herren und eifern ihnen ob ihrer Potentiale sogar nach. Wurde ursprünglich versucht, mit den Mitteln der Informatik die Struktur menschlichen Denkens zu simulieren, wurde der Computer nach und nach immer mehr selbst zum Modell von Intelligenz und Geist und kann so letztlich zum Ideal werden, das auch wir zu erreichen versuchen und dem wir, gelingt es uns nicht, sogar bereitwillig das Ruder überlassen.

\section{Perfektion oder Vollendung}

Diese kritischen Betrachtungen im Gefolge von Anders und Kierkegaard, die uns helfen können, die Faszination durch KI individual- und sozialpsychologisch besser zu verstehen, sollen nun nicht als Argumente gegen jeden technischen Fortschritt missverstanden werden. Dies betont etwa auch Papst Franziskus, der in seiner Enzyklika Laudato si' (LS) zweifellos als einer der schärfsten amtskirchlichen Kritiker technologischer Irrläufer in Erscheinung tritt. Diese Kritik entspringt nicht weltverneinender Maschinenstürmerei, sondern der Sorge vor einer Abkopplung technologischer Entwicklung vom Ziel der menschlichen Entfaltung und des gesellschaftlichen Gemeinwohls. ${ }^{23}$ Das Streben nach mehr Sicherheit, Lebensqualität, ja auch

22 Vgl. Dupuy, (2015), 57.

23 Vgl. Guggenberger (2016), 44-46. 
Bequemlichkeit ist sowohl aus ethischer wie aus theologischer Perspektive legitim und entspringt dem menschlichen Wesensmerkmal, über sich selbst und die jeweils gegebene Situation hinaus zu streben, in einer Form von Selbsttranszendenz, die Grundlage jeglichen Kulturschaffens ist.

Auf problematisches Terrain geraten wir allerdings dort, wo ein durch Kontingenz und innere Ambivalenz geprägtes Wesen versucht, sich selbst zu perfektionieren. Die Konzilskonstitution Gaudium et spes (GS) hält fest, dass der Mensch

$[\ldots]$ in sich selbst zwiespältig [ist]. Deshalb stellt sich das ganze Leben der Menschen, das einzelne wie das kollektive, als Kampf dar, und zwar als ein dramatischer, zwischen Gut und Böse, zwischen Licht und Finsternis. (GS 13)

Diese etwas esoterisch anmutende Redeweise wird durchaus lebenspraktisch greifbar, wenn es an anderer Stelle heißt:

Unter diesen Umständen zeigt sich die moderne Welt zugleich stark und schwach, in der Lage, das Beste oder das Schlimmste zu tun; für sie ist der Weg offen zu Freiheit oder Knechtschaft, Fortschritt oder Rückschritt, Brüderlichkeit oder Hass. Zudem wird nun der Mensch sich dessen bewusst, dass es seine eigene Aufgabe ist, jene Kräfte, die er selbst geweckt hat und die ihn zermalmen oder ihm dienen können, richtig zu lenken. (GS 9)

In eine destruktive Richtung werden die Weichen dort gestellt, wo Menschen dem Geschöpf und nicht dem Schöpfer dienen, wie es Paulus im Römerbrief ausdrückt (Röm 1,25). Dies führt uns zur theologischen Rede vom Götzendienst. Auch Günther Anders verweist auf diese Thematik unter Rückgriff auf Augustinus, für den die Verwechslung zwischen Schöpfer und Geschaffenem eine menschliche Grundversuchung ist. Dennoch meint Anders im kritisierten Götzendienst ein grundlegend anderes Problem zu erkennen als in der prometheischen Scham. Denn nun sei ja der Mensch der Schöpfer seiner Fabrikate. „Vertauschung von creator und creatum bedeutet hier daher, dass er [der Mensch] die Ehre, die er seinen Dingen erweist, eigentlich 
sich selbst schuldet und allein sich selbst.“24 Der Mensch mache sich daher nicht des Hochmuts schuldig, sondern der Selbstentwürdigung, die Anders an anderer Stelle allerdings selbst mit dem Götzendienst in Verbindung bringt, wenn er schreibt: „Solche Selbsterniedrigung vor Selbstgemachtem hat es seit dem Ende der Idolatrie nicht wieder gegeben." ${ }^{\text {25 }}$ Was Anders übersieht, ist, dass jener Götzendienst, den etwa die biblischen Propheten mit sarkastischer Kritik belegen, nicht in erster Linie in der Verehrung von Naturphänomenen besteht, sondern gerade darin, dass Menschen handwerklich-technisch ein Objekt herstellen, aufstellen und sich letztlich vor ihm niederwerfen, weil sie ihm eine eigenständige, ihnen selbst überlegene Wirksamkeit zuschreiben.

Ob sich aus dem Vergleich mit solch archaischen Phänomenen etwas für unseren Umgang mit modernsten Technologien lernen lässt, mag man in Zweifel ziehen. Das grundlegende menschliche Problem scheint mir jedoch tatsächlich das gleiche geblieben. Geht es doch immer darum, dass wir nicht fähig sind zu empfangen, es nicht ertragen können, uns selbst geschenkt zu sein, und damit im Letzten nie absolut selbstbestimmt und frei sind. Unser Streben nach Perfektion, sei es in intellektueller, moralischer oder physischer Form scheitert an uns selbst. Über die Enttäuschung darüber soll uns die Kreation perfekter Produkte hinwegheben, in denen wir ein Idealbild von uns selbst zu finden meinen, das aber nur Abbild sein kann, das somit entweder mit unseren Fehlern behaftet bleibt, oder ein auf wenige Aspekte dessen, was uns selbst ausmacht, beschränkter Abklatsch. Vollendung kann einem Wesen, das auf Beziehung ausgerichtet ist, nur geschenkt werden.

\section{Literatur}

Anders, Günther (1968), Die Antiquiertheit des Menschen: Über die Seele im Zeitalter der zweiten industriellen Revolution. München.

24 Anders (1968), 25.

25 Anders (1968), 30. 
Benedikter, Roland (2021), Technology: the new God? Techno-metaphysics and homo deus: contemporary attempts towards a radical perspective on the digital change of religion. In: Giulia Isetti / Elisa Innerhofer / Harald Pechlaner / Michael de Rachewiltz (Hg.), Religion in the Age of Digitalization. Kindle-Version. London, New York.

Dupuy, Jean-Pierre (2007), Die Ethik der Technologie im Zeitalter der dohenden Apokalypse. In: Wolfgang Palaver / Andreas Exenberger / Kristina Stöckl (Hg.), Aufgeklärte Apokalyptik: Religion, Gewalt und Frieden im Zeitalter der Globalisierung (Edition Weltordnung - Religion - Gewalt 1). Innsbruck, 229-249.

Dupuy, Jean-Pierre (2015), A Short Treatiese on the Metaphysics of Tsunamis. East Lansing.

Floridi, Luciano (2015), Die vierte Revolution. Wie die Infosphäre unser Leben verändert. Berlin.

$G S=$ Gaudium et spes (Über die Kirche in der Welt von Heute)

Zweites Vatikanisches Konzil (1980), Die pastorale Konstitution über die Kirche in der Welt von heute „Gaudium et spes“. In: Karl Rahner / Herbert Vorgrimler (Hg.), Kleines Konzilskompendium. Sämtliche Texte des Zweiten Vatikanums. 14. Auflage. Freiburg i. Br., 449-552.

Guggenberger, Wilhelm (2016), Zu viele Werkzeuge, zu wenig Ziele. Technikskepsis in der Enzyklika Laudto si'. In: Datterl, Monika / Guggenberger, Wilhelm / Paganini, Claudia / Siebenrock, Roman (Hg.), Papst Franziskus - ein erstes Resümee. Innsbruck, 39-58.

Harari, Yuval Noah (2017), Homo Deus. Eine Geschichte von Morgen. München.

Harari, Yuval Noah (2018), 21 Lektionen für das 21. Jahrhundert. München. Kehlmann, Daniel (2021), Mein Algorithmus und Ich: Stuttgarter Zukunftsrede. Stuttgart. Kindle-Ausgabe.

Kierkegaard, Sören (1983), Die Krankheit zum Tode (Gesammelte Werke, 24. und 25. Abteilung). 3. Auflage. Gütersloh.

Kreutzer, Ralf T. / Sirrenberg, Marie (2019), Künstliche Intelligenz verstehen. Wiesbaden.

Kurzweil, Ray (2001), Die Maschinen werden uns davon überzeugen, dass sie Menschen sind. Ein Gespräch mit Ray Kurzweil. In: Frank Schirrmacher (Hg.), Die Darwin AG. Wie Nanotechnologie, Biotechnologie und Computer den neuen Menschen träumen. Köln, 98-109. 


\section{Wilhelm Guggenberger}

Kurzweil, Ray (2005), The Singularity is near. When Humans transcend Biology. London, 2005.

$L S=$ Laudato si' (Über die Sorge für das gemeinsame Haus)

Franziskus (2015), Enzyklika Laudato si' von Papst Franziskus über die Sorge für das gemeinsame Haus. http://www.vatican.va/content/ francesco/de/encyclicals/documents/papa-francesco_20150524_enciclica-laudato-si.html [27. 5. 2020].

Metz, Karl Heinz (2006), Ursprünge der Zukunft. Die Geschichte der Technik in der westlichen Zivilisation. Paderborn.

Ortega y Gasset, José (1956), Betrachtungen über die Technik. In: José Ortega y Gasset, Gesammelte Werke. Band 4. Stuttgart, 32-95. 